Écrire

l'histoire

\section{Écrire l'histoire}

Histoire, Littérature, Esthétique

$11 \mid 2013$

Présent (1)

\title{
Darwinia, de Robert Charles Wilson
}

Une objectivation populaire de l'uchronie

Irène Langlet

\section{OpenEdition}

Journals

Édition électronique

URL : http://journals.openedition.org/elh/320

DOI : 10.4000/elh.320

ISSN : 2492-7457

Éditeur

CNRS Éditions

\section{Édition imprimée}

Date de publication : 15 mai 2013

Pagination : 133-142

ISBN : 978-2-35698-058-8

ISSN : 1967-7499

Référence électronique

Irène Langlet, «Darwinia, de Robert Charles Wilson », Écrire l'histoire [En ligne], 11 | 2013, mis en ligne le 15 mai 2016, consulté le 23 septembre 2020. URL : http://journals.openedition.org/elh/320 ; DOI :

https://doi.org/10.4000/elh.320 


\section{Darwinia, de Robert Charles Wilson \\ Une objectivation populaire de l'uchronie}

L'un des GRANDS DÉBATs de la narratologie porte sur les marqueurs de fictionnalité, et le récit historique en est l'un des arguments majeurs ${ }^{1}$, sans que les historiens y aient paradoxalement un si grand intérêt. Car en ce qui les concerne, il faut bien se rendre à l'évidence: toutes les manières d'écrire l'Histoire ${ }^{2}$, y compris l'appareil péritextuel, peuvent avoir leur version fictionnelle ${ }^{3}$. Dorrit Cohn elle-même fait une exception très coûteuse, en termes logiques, à sa thèse essentialiste, pour considérer des romans comme La Mort de
Virgile (1945), de Hermann Broch, ou les Mémoires d'Hadrien (1951), de Marguerite Yourcenar ${ }^{4}$. Plus pragmatique, la notion de « fiction flottante » de Jean-Marie Schaeffer articule les divers paramètres énonciatifs et narratifs de la «feintise ludique partagée » (définition censée clarifier la « fiction ») et ceux des " postures d'immersion variables », c'est-à-dire des diverses attitudes de partage de l'univers fictif adoptées par le lecteur ${ }^{5}$. Qu'en retenir lorsque l'on s'interroge sur l'écriture de l'Histoire à travers l'uchronie?

Irène Langlet, université de Limoges, EHIC - Espaces humains et interactions culturelles.

1. Pour une synthèse du débat théorique sur les marqueurs de fictionnalité, voir Frank Wagner, «Le récit fictionnel et ses marges: état des lieux », Vox Poetica, 2006; <www.vox-poetica.org/t/articles/wagner2006.html>, cons. 29 janv. 2013.

2. On donnera désormais une majuscule à la discipline (Histoire vs. Géographie), pour la distinguer du terme narratologique (histoire vs. récit).

3. Pour une synthèse des travaux linguistiques sur la question du récit historique, voir Bérenger Boulay, « Avec des "si” \& des "-rait". Comment on récrit l'Histoire », Acta Fabula, « Faire \& refaire l'histoire », <www.fabula.org/revue/document6409. php>, cons. 29 janv. 2013.

4. Dorrit Cohn, Le Propre de la fiction [1999], trad. de l'anglais par Claude Hary-Schaeffer, Éd. du Seuil (Poétique), 2001.

5. Jean-Marie Schaeffer, Pourquoi la fiction?, Éd. du Seuil (Poétique), 1999. 
Premièrement, l'ensemble de techniques dont la narratologie a effectué la mise en ordre (essentiellement sous l'impulsion de Gérard Genette). Les procédés de mise en valeur de l'événement, de tension narrative, d'identification des personnages, etc., constituent une boîte à outils commune pour l'historien et pour l'écrivain. La structure des topoï, en particulier, va au-delà de leurs thématiques; écrire la Grande Guerre n'implique pas les mêmes codes qu'écrire un bombardement de Londres ou l'exploration d'un continent mystérieux (pour prendre des exemples dans le roman dont il sera question ici).

Deuxièmement, l'importance des conditions de production, de réception et de circulation du récit: Schaeffer invite bien à une théorie critique fondée sur un faisceau de critères et non (comme l'espère Cohn) sur une essence illusoire de la fiction. Ainsi, lire en français, en France, un récit publié aux ÉtatsUnis en 1998 revisitant l'Histoire depuis 1912 implique un brassage intense des référents du récit: le contrefactuel n'est pas spontanément identifié de la même manière ici et là, et la frontière entre les référents attestés et les pseudo-référents suit là un parcours bien plus sinueux qu'ici. Or ce parcours caractérise précisément l'acte de lecture uchronique, et intéresse donc à la fois l'historien et le littéraire.

Le débat sur les marqueurs de fictionnalité, en effet, et troisièmement, est riche de ce qu'il n'arrive pas à trancher, comme on l'entend bien, a contrario, dans un avis comme celui-ci:

La fermeté des arguments proposés par [Dorrit] Cohn contre le «tout fictionnel » ou le «tout subjectif » des déconstructions «postmodernes » du récit $(\mathrm{au}$ nombre desquelles [Dorrit] Cohn compte certaines positions de [Gérard] Genette) l'emporte sans doute sur l'inconvénient de masquer les fructueux échanges qu'entretiennent avatars fictionnels et non fictionnels d'un même genre (par exemple en matière de biographie) ou les interactions complexes entre auteur, genre et lecteur qui sont le propre de certaines poétiques romanesques contemporaines. ${ }^{6}$

C'est le cas dans Darwinia, de Robert Charles Wilson ${ }^{7}$, que l'on pourrait qualifier de métauchronie aussi bien que de « roman de genres » (pluriel délibéré). On montrera en quoi dans une lecture cursive, orientée par trois objectifs: identifier les points de « fabrique de l'uchronie », adossés à une véritable combinatoire de référents culturels, ce qui permet par ricochet de déceler les points de « fabrique de l'Histoire »; observer, au fil des passages métatextuels fort nombreux ici, les indices permettant de définir l'uchronie comme pratique de dérèglement référentiel triplement contrôlée: par la discipline historienne, par la démarche cognitive d'apprentissage, par la poétique du narratif; proposer enfin quelques conclusions envisageant comment la lecture d'une

6. Alexandre Gefen, «Le partage des eaux », Acta Fabula, 2000, <www.fabula.org/revue/cr/6.php>, cons. 29 janv. 2013.

7. Robert Charles Wilson, Darwinia [1998], trad. de l'anglais (Canada) par Michèle Charrier, Denoël, 2000. Édition utilisée: Gallimard (Folio SF), n¹51, 2003. 
telle uchronie permet d'éclairer l'écriture, et la lecture, de l'Histoire et de son archive.

\section{Un roman de genres}

Darwinia raconte comment Guilford Law, photographe de Boston, se joint à une expédition d'exploration du «nouveau monde », ou Darwinie, constitué du continent européen coupé du reste de la planète par un phénomène cosmique mystérieux survenu en 1912, et comment ce qu'il y découvre entraîne sa vie entière dans un combat contre des extraterrestres hostiles. L'événement est narré dès le prologue (situé en 1912) sous les deux points de vue du jeune Guilford adolescent et de l'équipage confronté au monde préhistorique qu'est devenue l'Angleterre. Ce prologue exploite les codes du Bildungsroman réaliste et du roman d'aventures en mer, dans un double mouvement d'exposition d'un mystère et de positionnement face à lui :

Car l'inacceptable était là. Il n'y avait pas trace de la ville. La côte n'avait pas été aménagée. À la place des rues de Queenstown - des exportateurs, des grues, des dockers, des émigrants irlandais - ne s'étendait qu'une forêt sauvage enveloppant un rivage rocailleux. ${ }^{8}$

Pour [Guilford], il ne s'agissait pas d'un miracle, juste d'un mystère. Inexplicable, mais peut-être pas intrinsèquement tel. ${ }^{9}$
L'horizon d'attente du roman est bien celui d'une uchronie, c'est-à-dire d'un monde imaginaire traité comme réel, qui découle d'une disjonction historique bien identifiée. Le roman d'aventures et d'exploration d'île codifie aussi les rapports réalistes entre les parties connues et inconnues du monde. Seule une discrète touche de fantastique, à l'occasion de la description de plantes géantes, pose le premier indice du genre « roman de monde perdu » qui se développera ensuite.

Les quatre parties du roman abordent successivement trois étapes de la vie de Law. Les livres 1 et 2 (1920-1921), qui retracent l'expédition et ses découvertes très énigmatiques, se terminent sur un combat contre des entités probablement extraterrestres, dans le contexte de conflits politiques entre les restes des empires coloniaux. Huit années après 1912, voici brossée l'uchronie: - Londres était autrefois la plus grande cité du monde. Le saviez-vous, Mrs. Law? Rien de comparable avec l'ébauche grossière qu'elle est à présent. Des cabanes, des cagibis, de la boue. Mais je regrette de ne pas pouvoir vous montrer Copenhague. Ça, c'était une ville! Une ville civilisée. ${ }^{10}$

Le récit de l'exploration de la Darwinie exploite les principes du roman d'aventures dans sa variante, très populaire dans les années 1910-

\footnotetext{
8. Ibid., p. 20.

9. $\quad$ Ibid., p. 31.

10. Ibid., p. 41.
} 
$1920^{11}$, du «monde perdu » - voyez Conan Doyle en 1912 (précisément):

Il découvrit un marécage à la végétation épaisse balayé par le vent d'ouest - les marais salants de la large embouchure rhénane. Les stromatolithes s'y dressaient tels des monuments surnaturels, et les arbres-flûtes avaient envahi le delta partout où le limon était assez épais pour le réseau de leurs racines. ${ }^{12}$

Les coïncidences de ces dates ne sont évidemment pas un hasard, et les évocations explicites des grands auteurs populaires (Herbert George Wells, Jules Verne, Edgar Rice Burroughs, mais jamais Conan Doyle) dans le cours même de la diégèse le suggèrent au lecteur:

« La planète rouge », commenta Guilford, résumant ainsi tout ce qu'il en savait - outre le fait qu'elle possédait deux lunes et avait fourni à Burroughs ainsi qu'à Wells, un Anglais, matière à quelques œuvres de bon aloi. ${ }^{13}$

Un scénario « contre-uchronique » émerge par petites touches, d'abord adossé aux codes du roman fantastique et d'horreur (rêves étranges, cité maudite, morts réincarnés dans des humains possédés):

Parce que tu es mort, déclara le dieu fantôme.

Ce qui n'avait rien de rassurant.

Ce n'est pas vrai, protesta le spirite.

Tu t'es noyé dans l'Atlantique en 1917, quand un transport de troupes américain a été coulé par une torpille allemande. ${ }^{14}$
Avant de se fixer en roman de guerre:

Il se rappelait le bois Belleau et Bouresches. Le champ de blé rouge de coquelicots. Tom Compton, coupé en deux par une rafale de mitrailleuse. Il se rappelait le chagrin. ${ }^{15}$

Le livre 3 (1945) narre un nouveau combat contre les mêmes entités, avec l'aide d'un personnage issu d'une Histoire alternative du monde imaginaire - équivalant, pour le lecteur, à l'Histoire attestée - que j'appelle contre-uchronie. Dans cette dernière, la Première Guerre mondiale a lieu; le personnage se présente comme le double immortel de Guilford Law dans cet univers parallèle. Sur fond d'uchronie, stabilisée par l'écriture réaliste, se détachent non seulement la contre-uchronie, mais aussi les autres codes investis par le roman: roman de guerre, roman d'horreur dans la lutte contre les entités monstrueuses, et roman de science-fiction cosmique. Entre chacune des parties s'intercale en effet un "Interlude » précisant peu à peu, en rupture radicale avec le reste du roman, une guerre cosmique dans laquelle l'humanité n'est qu'un pion (scénario de « complots d'Immortels galactiques » bien connu de la science-fiction):

Des entités semi-conscientes - des codes parasites évolutifs autoreproducteurs, dissimulés dans l'entrelacs des

11. Pierre Benoît reçoit le Grand Prix du roman de l'Académie française pour L'Atlantide en 1919.

12. Robert Charles Wilson, op. cit., p. 89.

13. Ibid., p. 147.

14. Ibid., p. 137.

15. Ibid., p. 309. 
signaux de Higgs circulant entre les galaxies - s'étaient emparées des protocoles structurels des Archives, lesquelles perdaient à chaque seconde qui passait de l'information, sans espoir de la récupérer.

Qui pis était, l'information restante se transformait. ${ }^{16}$

Ce charabia n'est nullement à prendre au sérieux, comme l'indique cet incident dialogue ironique du personnage avec son double immortel :

- Nous nous trouvons à l'intérieur des Archives, déclara son compagnon. Plus précisément, dans une suite logique nodulaire attachée aux protocoles opératoires de l'ontosphère terrestre.

- Tout s'explique.

- Désolé. Ce que je voulais dire, c'est que nous sommes toujours dans les Archives [... ${ }^{17}$

Le livre 4 (1965) donne le combat final, et enfin victorieux, après lequel le vieux Law est décrit comme au seuil d'une mort apaisée.

Ce faisceau générique, loin de compliquer la tâche d'une lecture attentive à la « fabrique de l'uchronie », me semble éclairer au contraire la manière dont un environnement narratif permet de solliciter, ou bien de désactiver, le réflexe uchronique. Ainsi, la découverte, au cœur de ce qui fut l'Allemagne, d'une cité abandonnée composée d'énormes blocs de basalte n'engendre pas d'hypothèse uchronique; enchâssée dans le synopsis d'exploration du monde
16. Ibid., p. 177.
17. Ibid., p. 366.
18. Ibid., p. 122.
19. Ibid., p. 15-16.

perdu, elle débouche, en toute logique de genre littéraire, sur les révélations extraterrestres. Une allusion aux romans martiens de Burroughs vient insister sur cette logique de genre, au cas où on ne l'aurait pas décelée:

- Les bêtes sont intéressantes, observa Guilford.

Comme les thoats des romans de Burroughs. Les montures martiennes. ${ }^{18}$

\section{Une fictionnalité différentielle}

En revanche, dans le prologue et le livre 1, une poétique réaliste incite le lecteur à une intense activité encyclopédique pour faire le départ entre les références attestées et celles qui ne sont que feintes:

C'était l'époque des navires fabuleux: la Great White Fleet, les vaisseaux de ligne de la Cunard et de la White Star, les monstruosités de l'Empire britannique, tels le Teutonic ou le Mauretania. [...] l'Oregon, un bâtiment de ligne plus tout jeune de la White Star, parti de New York à destination de Queenstown et de Liverpool. ${ }^{19}$

En outre, les notes du traducteur (NdT) mettent en évidence la reconnaissance des références historiques - ou non. Ce hiatus culturel bien connu des traductologues revêt ici une importance considérable, puisque le genre uchronique tout entier est dépendant de la combinatoire des éléments reconnus et de ceux qui ne le sont pas. Ce tableau à $n$ dimensions est compliqué par la 
subdivision culturelle des aires linguistiques: un Canadien de Toronto (ville où réside l'écrivain, d'origine américaine), un Québécois, un Français ou un Belge n'auront pas besoin des mêmes « NdT » pour construire leur uchronie.

Mais l'ignorance du lecteur français fait planer le doute sur les connaissances du lecteur autochtone. D'ailleurs, Wilson écrit-il pour le public canadien ou américain? Quelles sont leurs références historiques? Et, à la réflexion, les aires nationales ou linguistiques sont-elles le meilleur cadre d'identification de ces encyclopédies ${ }^{20}$ ? Dans l'exemple donné plus haut, le passionné de marine à vapeur ne subsume-t-il pas le lecteur anglais, français, ou américain? Dans celui-ci :

Ne leur parvinrent ni C.Q.D. ni S.O.S. - le nouveau signal de détresse -, ni appels dramatiques de navires en train de sombrer. ${ }^{21}$

Ou devant ce Teutonic, méconnu, paronyme du célèbre paquebot sinistré - comme par un fait exprès - en avril 1912 (et que le roman n'évoque jamais), le lecteur ignorant tout de l'histoire du morse ou de celle des grands transatlantiques n'est-il pas perversement incité à faire des hypothèses uchroniques? À moins qu'il ne soit (autre protocole de lecture) incité à compulser des documents d'Histoire pour augmenter ses connaissances. Il y aurait là un effet didactique collatéral de l'uchronie, faisant de sa lecture ambivalente le support d'une activité non fictionnelle... et peut-être d'une «fabrique de l'Histoire », par ricochet.

La fabrique de l'uchronie engage donc non seulement un «point de disjonction » à partir duquel une Histoire contrefactuelle sert de cadre référentiel à un récit réaliste (ici, le " miracle » de 1912), mais aussi une collection de référents qui hiérarchise des niveaux de fictionnalisation: référents historiques et connus ; référents historiques et méconnus ou relevant de domaines spécialisés; référents uchroniques feints. Et ces niveaux ne se succèdent pas sagement selon une progression logique, faisant ainsi passer le lecteur d'une fiction adossée à l'Histoire à une fiction qui en serait complètement déconnectée; le va-et-vient constant entre la fiction et le référent historique implique plutôt un mode de fictionnalité différentiel. C'est ce que viendrait confirmer un dernier aspect, sans doute le plus spectaculaire, du genre uchronique (et l'on entend bien ici le genre romanesque, et non la pratique contrôlée des Histoires contrefactuelles $\left.{ }^{22}\right)$ : la permanence d'une ligne historique référentielle,

20. J'entends ce mot au sens sémiotique d’Umberto Eco, comme ensemble des connaissances nécessaires à l'interprétation pendant la lecture.

21. Robert Charles Wilson, op. cit., p. 16.

22. Voir Richard Saint-Gelais, "Quelques avatars de l'advenu: excursions en uchronie », dans L'Empire du pseudo. Modernités de la science-fiction, Québec, Nota Bene (Littérature(s)), 1999, p. 45-89. 
même et surtout après le point de disjonction, comme si l'intérêt romanesque tenait autant à la disjonction qu'à un "rattrapage » de l'Histoire attestée à travers l'Histoire revisitée. Darwinia en offre une saisissante objectivation, en écho aux divers dispositifs rivalisant d'ingéniosité que peuvent mettre en place Philip K. Dick, Christopher Priest ou Roland C. Wagner.

L'événement imaginaire de ce roman se caractérise pourtant par une absence totale de subtilité. La partition du monde en deux zones est mise en scène comme un miracle cosmique; l'explication de ce miracle est renvoyée à des extraterrestres omnipotents. On est loin des ajustements minutieux permis par quelques années de différence dans la mort d'un empereur, par exemple ( $L a$ Reine des lumières, de Xavier Mauméjean, 2009). Le résultat, on l'a vu, est un patchwork de codes romanesques dont l'intrication permet de comprendre comment le réflexe de lecture uchronique est ici mis au service d'une plus vaste quête romanesque. L'ultime horizon de cette dernière est une sorte d'Armageddon entre les êtres cosmiques destructeurs et les êtres humains soucieux de stabiliser le cours de l'Histoire, et de préserver les «Archives cosmiques » contenant l'infinité de tous les mondes possibles de tous les temps. Comment ne pas voir ici une formulation, grossière mais juste, du principe contrefactuel tel qu'enrôlé par la littérature? Darwinia utilise, pour cette formulation métapoétique, les outils de la littérature de genre la plus populaire: mondes perdus, aven- tures préhistoriques, dieux cosmiques malfaisants - et opportunément insectiformes - prenant possession d'êtres humains, combats à l'arme lourde fortement homicides. Mais ce tourniquet des genres permet de mettre en valeur la confrontation des éléments uchroniques et contre-uchroniques: en incarnant chaque ligne historique dans une version de Guilford Law, et en objectivant dans la diégèse leur compagnonnage progressif. D'abord amené par les codes du récit fantastique (rêves, thème du double), le Guilford contre-uchronique (c'est-à-dire placé dans une Histoire qui est la nôtre) se manifeste de plus en plus fraternellement au Guilford uchronique, jusqu'au dernier chapitre, où ce dernier choisit d'avoir un destin mortel, contrairement à l'autre. La signification en est clairement métaromanesque, et donne aux personnages une valeur allégorique. Regrette-ton la virtuosité du retournement métaleptique d'un Dick, ou des effets de chatoiement diégétique d'un Priest? Là encore, l'incarnation dans ces deux destins de la permanence de l'Histoire réelle face à la fugacité des histoires fictionnelles ne s'embarrasse pas de subtilité. Mais elle affirme la règle du jeu uchronique avec bon sens. Et, peutêtre, pédagogie.

\section{Les récompenses cognitives du lecteur d'uchronie}

Je suis tentée, en effet, de verser cette réaffirmation au compte des effets collatéraux de l'écriture uchronique en général, et de ce roman en parti- 
culier. Car si l'uchronie est un usage contrôlé du dérèglement de l'écriture de l'Histoire, on peut déceler ce contrôle dans au moins trois dynamiques complémentaires activées pendant la lecture: d'abord une dynamique littéraire, soutenue par toutes les variantes populaires de la tension narrative; ensuite, une dynamique historienne, alimentée par le plaisir du repérage des référents historiques. Combinés, ces deux mouvements permettent de prendre la mesure des codes d'écriture, ceux des genres populaires bien sûr, mais aussi ceux des topoï historiens: Grande Guerre évidemment, mais aussi émeutes urbaines du premier $\mathrm{Xx}^{\mathrm{e}}$ siècle aux ÉtatsUnis, crise de 1929:

Par la suite - durant l'époque troublée où miséreux et sans-abri devinrent si dramatiquement nombreux, où charbon et pétrole se firent si coûteux, où des émeutes éclatèrent car la population réclamait du pain $[\ldots] .{ }^{23}$

Le frottement permanent des deux lignes historiques permet également de détourner ironiquement des thèses discutées:

Les États-Unis avaient déclaré avec arrogance que l'Europe devait être « librement ouverte aux colons et dépourvue de frontières »- la prétendue doctrine de Wilson, qui impliquait en pratique l'hégémonie américaine, un nouveau monde américain. ${ }^{24}$

Cette fictionnalité différentielle éclaire surtout, dans ses combinaisons de thèmes et de codes d'écriture, la façon dont se construisent des symboles historiques: Londres bombardée, une émeute dans Johannesburg coloniale, les réfugiés d'une guerre russo-japonaise... Autant d'indicateurs de l'imaginaire collectif dont le roman montre le caractère construit, narré, et éminemment réversible en fonction du contexte. Faut-il en conclure que l'uchronie est un genre de la déconstruction culturaliste postmoderne et du subjectivisme généralisé, dont l'engouement s'appuie sur l'impossibilité structurelle de désigner des marqueurs de fictionnalité?

J'y verrais plutôt une pratique ludique, certes, mais dont le jeu consiste surtout à scénariser la dynamique cognitive que j'identifierais ici comme le troisième mouvement de contrôle du dérèglement de l'écriture de l'Histoire. Si on admet qu'il est indispensable d'y discriminer le factuel et le contrefactuel, l'uchronie ne saurait être lue comme une simple aventure en monde imaginaire. Engageant tout à la fois une mise à l'épreuve de la fiction et une mise à l'épreuve de l'Histoire, ce genre serait à l'acquisition de connaissances historiques postmoderne ce que le roman historique fut à l'époque moderne. Lors de celle-ci, la maîtrise des codes littéraires et historiens a pu emprunter la voie d'une parfaite et géniale dissimulation de ces derniers; elle emprunte plutôt, dans celle-là, la voie d'une confrontation productive, rusée parfois, grossière souvent.

23. Robert Charles Wilson, op. cit., p. 28.

24. Ibid., p. 100-101. 
Mais c'est à l'évidence un canal d'acquisition de connaissances, dont les amateurs auront tôt fait de chercher à vérifier si, effectivement, « l'été 1920 fut froid, du moins à Washington ${ }^{25}$ ", ou s'il y eut bien une émeute en Afrique du Sud en $1912^{26}$. Il faudrait ici mesurer le spectre des pratiques de lecture, de la plus naïve à la plus raffinée, de la plus hâtivement consommatrice à la plus méticuleusement vérificatrice; la hiérarchisation des niveaux de fictionnalisation évoquée plus haut accompagne, voire encode dans le texte cette variabilité des lectures.

À ce titre, la récompense cognitive préparée pour le lecteur passionné d'Histoire et de littérature populaire est à la hauteur, dans Darwinia, de l'effort qui lui est demandé. Car s'il est à la portée de n'importe quel lecteur de science-fiction uchronique (cible sans ambiguité de l'appareillage éditorial du roman, aux ÉtatsUnis comme en France) de reconnaître la comète de Halley, le pulp Argosy ${ }^{27}$ ou le National Geographic ${ }^{28}$, il est plus difficile d'identifier tel obscur homme politique américain opposé à Wilson ${ }^{29}$, d'apprécier le plaisant retournement

25. Ibid., p. 133.

26. Ibid., p. 61.

27. Ibid., p. 43, passim.

28. Ibid., p. 123.

29. Ibid., p. 135.

30. Ibid., p. 114.

31. Ibid., p. 238.

32. Conan Doyle, The Lost World, Londres, Hodder and Stoughton, 1912.

33. Robert Charles Wilson, op. cit., p. 160.
d'Einstein en mathématicien ${ }^{30}$, ou du vénérable Times réduit à " un quotidien de six pages imprimé sur du papier fibreux tiré d'arbresmosquées $^{31}$ ». Mais il reviendra aux seuls passionnés de repérer, dans l'intertexte littéraire, le roman caché dont Darwinia effectue la redécouverte, en même temps qu'il s'en inspire. Car, à la manière de la lettre volée d'Edgar Poe, ce roman est à la fois exhibé et dissimulé, sous une série de petits pièges citationnels : à côté des noms aisément reconnaissables de Wells, Verne, ou Lewis Carroll, la Darwinie est qualifiée de « continent perdu », ce qui appelle bien entendu le « monde perdu » de Conan Doyle ${ }^{32}$, lequel toutefois n'est jamais cité; les descriptions de paysages, les animaux préhistoriques se chargent de faire le lien. La référence explicite est celle d'Edgar Rice Burroughs - le créateur de Tarzan, du Cycle de Mars ou de Pellucidar-, convoqué avec insistance, mais jamais pour le titre exact, qu'il faudrait citer. Il se voit ainsi attribuer un livre imaginaire, Le Royaume perdu de Darwinie ${ }^{33}$, qui sert littéralement de guide au héros: 
Les contes imaginés par Mr. Burroughs sont un véritable Baedeker de ce continent! ${ }^{34}$

C'est fort habilement dissimuler l'hypotexte réel de Darwinia: un petit roman méconnu de Burroughs intitulé Beyond Thirty ${ }^{35}$, publié en revue en 1916, jamais traduit en français, auquel ses rééditeurs attribuèrent brièvement (de 1963 à 2001) le titre de The Lost Continent - des films synonymes recueillent de gros succès dans les mêmes années ${ }^{36}$. Dans ce roman, un explorateur de l'année 2137 s'aventure dans le monde sauvage qu'est devenue l'Europe après avoir été isolée du reste du monde à la suite de la Grande Guerre.

L'absence étonnante de mentions de ce livre dans les recensions de Darwinia suffirait à montrer le caractère facultatif de sa reconnaissance par les lecteurs. Wilson développe ainsi une histoire littéraire parallèle, sorte d'uchronie philologique, dontle plaisir du texte demande une culture poussée et la discrimination attentive des titres, œuvres et produits dérivés de l'industrie burroughsienne. J'y vois le dernier soulignement, astucieux celuici, de la portée critique que Darwinia exploite dans le genre uchronique. Critique créative, assurément, appuyée le plus souvent à des moyens didactiques rudimentaires comme l'allégorie ou l'objectivation diégétique des « deux Histoires » manipulées par le genre. Mais l'encodage d'une lecture plus virtuose, érudite et populaire à la fois, est bien présent; ce qui met ce roman en bonne place dans un corpus méta-uchronique, à côté du Maître du Haut Château (Philip K. Dick, 1962) ou de La Séparation (Christopher Priest, 2002).

34. Ibid., p. 217.

35. J'exprime toute ma gratitude à Matthieu Letourneux pour son aide dans ce repérage.

36. Sam Newfield, The Lost Continent, Lippert Pictures, 1951, 83 min, adaptation du roman de Conan Doyle; Michael Carreras, The Lost Continent, Warner-Pathé, 1968, 91 min, adaptation de Uncharted Seas, de Dennis Wheatley. 